proteins lack the conserved catalytic residues that are required for target cleavage, indicating that these proteins might require accessory factors to mediate target-mRNA turnover.

The authors proposed a two-step model for RNAi, which can explain how RNA-silencing pathways can achieve both specificity and amplification; exogenous and endogenous RNAi pathways involve functionally and structurally distinct AGO proteins, which function sequentially on primary and secondary siRNAs to direct gene silencing.

The researchers also found that some of the AGO proteins were required for development; depletion of $c s r$ - 1 resulted in defects in chromosome segregation and RNAi, depletion of $p r g-1$ resulted in germline defects, whereas ergo- 1 deficiency was associated with increased sensitivity to RNAi. The precise functions of AGO proteins in these processes require further investigation.

Ekat Kritikou

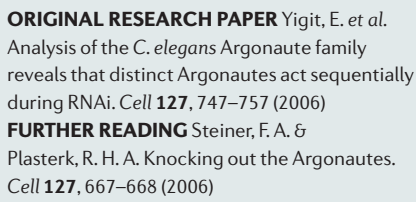

levels did not undergo apoptosis. Therefore, cells seem to have a semiefficient mechanism that can sense inappropriately high signalling levels and then induce apoptosis.

So, just as a mother might reward or punish her children to make them share, a mother cell uses a double mechanism to ensure the equal sharing of its signalling components between daughter cells: endosomes that contain crucial signalling molecules (including Tkv, as recruited by Sara) are equally distributed during mitosis, and signalling-component abnormalities can be sensed and handled by apoptosis.

Asher Mullard

ORIGINAL RESEARCH PAPER Bökel, C. et al. Sara endosomes and the maintenance of Dpp signaling levels across mitosis. Science $\mathbf{3 1 4}$, 1135-1139 (2006)

FURTHER READING Knoblich, J. A. Sara splits the signal. Science 314, 1094-1095 (2006)

MECHANISMS OF DISEASE

\title{
Cure for a broken heart?
}

Ischaemic damage that results from vascular insufficiency is a frequent cause of cardiac failure. Riley and colleagues now identify thymosin $\beta 4$ (T $\beta 4)$, a protein required for actin reorganization, as being essential for all key aspects of coronary vessel development in mice, and demonstrate that T $\beta 4$ stimulates the mobilization of vascular progenitors from adult epicardium.

To investigate the role of T $\beta 4$ in heart development, Riley and colleagues generated mouse embryos with a heart-specific $T \beta 4$ deficiency, designated T $\beta 4 \mathrm{sh}^{\mathrm{Nk}}$. $\mathrm{T} \beta 4 \mathrm{sh}^{\mathrm{Nk}}$ embryos displayed epicardial defects by embryonic day 12.5 (E12.5). By E14.5, these were accompanied by defects in the ventricular myocardium and by E16.5, severely affected embryos were dying.

\section{...T $\beta 4$ and AcSDKP function as} potent stimulators of coronary vasculogenesis and angiogenesis, offering protection following cardiac injury.

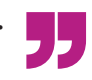

A lack of immunostaining for the endothelialspecific receptor TIE2 in the myocardium of $\mathrm{T} \beta 4 \mathrm{sh}^{\mathrm{Nk}}$ hearts signified the absence of microvessels. By contrast, TIE2 was strongly expressed in aberrant epicardial nodules, which indicates that these nodules represent a population of epicardium-derived cells (EPDCs) that have attempted, but failed, to migrate through the myocardium to form coronary vessels. Moreover, in T $\beta 4 \mathrm{sh}^{\mathrm{Nk}}$ hearts, smooth-muscle cells (which are also derived from EPDCs) failed to migrate into the myocardium to provide support to the coronary vessels. So, $T \beta 4$ knockdown in the heart leads to defects in epicardial cell migration and coronary vessel development.

These defects were non-cell autonomous, which indicates a loss of functional secreted $\mathrm{T} \beta 4$ and impaired paracrine signalling to the epicardium. Riley and colleagues therefore investigated the effect of soluble T $\beta 4$ on epicardial explants in vitro. Explants from wildtype embryonic hearts treated with $\mathrm{T} \beta 4$ showed significantly increased outgrowths of smooth-muscle cells and TIE2expressing endothelial cells. So, $\mathrm{T} \beta 4$ promotes vascular progenitor proliferation from embryonic epicardium - but can this be translated to a role for $\mathrm{T} \beta 4$ in angiogenic therapy for coronary artery disease?

Treatment of adult epicardial explants with $\mathrm{T} \beta 4$ stimulated extensive outgrowth of cells that differentiated into endothelial, smooth-muscle and fibroblastic cells. Remarkably, T $\beta 4$-treated adult EPDCs displayed a state of pluripotency equivalent to their embryonic precursors. The authors suggest that these cells could provide a source of endothelial and smoothmuscle vascular precursors for vascular regeneration in the ischaemic heart.

But what are the mechanisms that underlie the vasculogenic function of T $\beta 4$ ? The role of $\mathrm{T} \beta 4$ in actin reorganization could help promote EPDC migration. In addition, $T \beta 4$ is subject to proteinase activity, which results in the pro-angiogenic tetrapeptide $\mathrm{N}$-acetyl-serylaspartyl-lysyl-proline (AcSDKP). The level of AcSDKP was lower in T $\beta 4$-mutant hearts. And although injection of AcSDKP was unable to rescue the T $\beta 4$-mutant phenotype, it significantly enhanced endothelial cell differentiation from adult epicardially derived precursor cells. The authors conclude that, together, T $\beta 4$ and AcSDKP function as potent stimulators of coronary vasculogenesis and angiogenesis, offering protection following cardiac injury.

Rebecca Robey

ORIGINAL RESEARCH PAPER Smart, N. et al. Thymosin $\beta 4$ induces adult epicardial progenitor mobilization and neovascularization. Nature 15 Nov 2006 (doi:10.1038/nature05383) WEB SITE

Paul Riley's laboratory: http://www.ich.ucl.ac.uk/ich/ academicunits/MMU/CustomMenu01 